\title{
Cell-Material Interaction
}

\section{S04-01}

\section{Biocompatible nanoparticles with a polypeptide shell by emulsion polymerisation}

\author{
*M. Klapper ${ }^{1}$, F. Karagöz ${ }^{1}$, S. H. Parekh ${ }^{1}$, R. Dorresteijn ${ }^{1}$, K. Müllen ${ }^{1}$ \\ ${ }^{I}$ Max-Planck Institute for Polymer Research, Mainz, Germany
}

For nanoparticles that are used as intravascular drug delivery systems, aggregation resulting in carrier sizes $>250 \mathrm{~nm}$ is a serious issue as those systems are removed from the blood stream by Kupffer cells.[4] Herein, we describe the synthesis of polylactide particles decorated by a polyglutamic acid corona by non-aqueous emulsion polymerization. While in many other approaches polypeptides are either after particle formation adsorbed or grafted herein we us a specially designed emulsifier to form a polypeptide shell.

A light sensitive PEG-block-poly((1-pyrenyl methyl) glutamate) (PEG- $b$-PGlu(Pyr)) copolymer is synthesized and used as emulsifier in a nonaqueous emulsion polymerization of lactide which is required due to the moisture sensitivity of the polymerization catalysts. Poly(L-lactide) (PLLA) nanoparticles were synthesized via ring-opening polymerization of L-lactide with a moisture-sensitive catalyst in a non-aqueous emulsion consisting of acetonitrile, cyclohexane, and the PEG-b-PGlu(Pyr) copolymer as emulsifier. Upon UV irradiation, hydrophobic pyrenyl methylene units are cleaved from the block copolymer, resulting in a polarity reversal of the particle surface from hydrophobic to hydrophilic. The product particles have a fully hydrophilic and biocompatible PEG- $b$-PGlu shell and can be dispersed in water without aggregation. Furthermore, introducing MMP-3 cleavable peptide sequences in the nanoparticles allows for a full degradation of the particles when they are getting close to tumor cells. This offers the opportunity to selectively release drugs which have already been incorporated before during in the particle formation.

The particles consist exclusively of non-toxic biodegradable polymes (polylactide and polypeptide making them suitable candidates for medical applications in particular for cancer therapy.

[1] Polarity Reversal of Nanoparticle Surfaces by the Use of Light-Sensitive Polymeric Emulsifiers R. Dorresteijn, R. Ragg, G. Rago, N. Billecke, M. Bonn, S. H. Parekh, G. Battagliarin, K. Peneva, M. Wagner, M. Klapper, K. Müllen, Biomacromolecules 2013, 14, $1572-1577$.

[2] Biocompatible Polylactide-block-Polypeptide-block-Polylactide Nanocarrier, R. Dorresteijn, N. Billecke, S. H. Parekh, M. Klapper, K.Müllen, J. Pol. Sci, Part A: Pol. Chem 2015, 53, 200

Figure 1

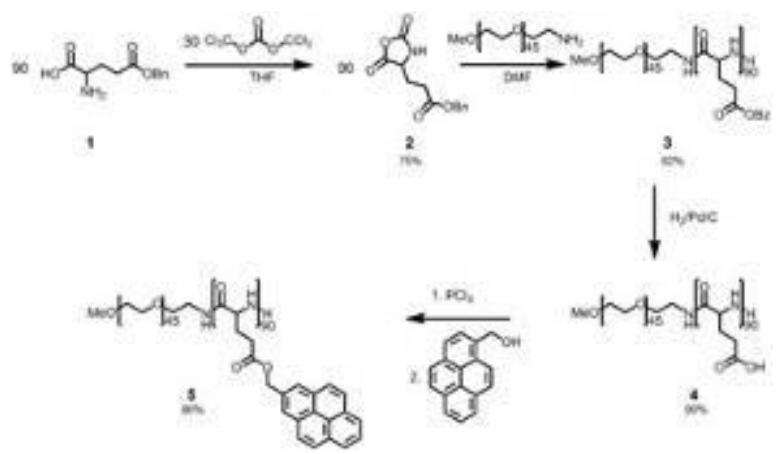

Figure 2

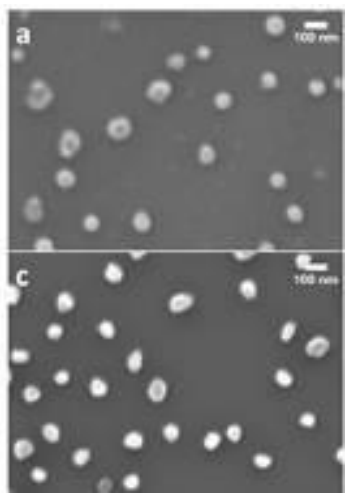


S04-02

\section{Tailormade Polymer Coatings for Microsystems and Engineered Biointerfaces}

*J. Rühe ${ }^{1}$

${ }^{1}$ University of Freiburg, Department for Microsystems Engineering, Freiburg, Germany

\section{Introduction}

All interactions of materials with their environments are controlled by their surfaces. In many cases, materials which are perfect for device generation will not be compatible with a biological environment. Thin polymer layers can tailor the surface properties precisely to the specific needs. However, they need to be stable and must not dissolve or delaminate during use. Chemistries for the attachment of the polymers to the surfaces are needed.

\section{Objectives}

In the presentation a new strategy will be presented which allows to generate micropatterned polymer coatings with tailor-made properties on different substrates (oxides, polymers, tissues).

\section{Materials \& methods}

The coatings are generated from reactive prepolymers. Upon deposition and activation a $\mathrm{C}, \mathrm{H}$ insertion leads to crosslinking and surfaceattachment. The films are characterized by means of surface analysis, the adsorption of biomolecules are followed by SPR. Fluorescence microscopies are used for blood compatibility tests.

\section{Results}

Polymers bearing reactive groups which connect to neighboring $\mathrm{C}, \mathrm{H}$-groups $(\mathrm{C}, \mathrm{H}$ insertion crosslinking, $\mathrm{CHIC})$ yield surface-attached polymer networks on different materials. Standard microlithographic techniques can be applied to generate two and three dimensional surface architectures.

Hydrogel coatings show an anisotropic swelling behavior which makes them protein and cell repellent. Such surfaces are bioinert even for extended periods of time. Such coatings can be used in blood contacting environments and for the reduction of scar formation after a glaucoma treatment. The combination of a bioinert background and specific probes leads to precise and reliable bioanalytical devices.

\section{Conclusion}

Surface attached hydrogels are stable and bioinert surface coatings with excellent cell repellency. Possible applications range from blood compatible surfaces to bioanalysis. 
Abstracts - Annual Meeting of the German Society for Biomaterials 2018 - Braunschweig, November 08 - 10 • DOI 10.1515/bmt-2019-7004

Biomed. Eng. - Biomed. Tech. 2019; 64(s1): p30-p35 • @ by Walter de Gruyter • Berlin • Boston

S04-03

\title{
$3 T 3$ fibroblast interaction with 3D-nanofibrous collagen scaffolds
}

\author{
*N. Suter ${ }^{1}$, S. Stebel ${ }^{1}$, D. Marx ${ }^{1}$, D. Brüggemann ${ }^{1}$ \\ ${ }^{1}$ University of Bremen, Institute for Biophysics, Bremen, Germany
}

Collagen is the main component in the nanofibrous extracellular matrix (ECM). It has previously been processed into nanofibers by electrospinning [1], self assembly [2] or extrusion [3]. For the first time, we combined self assembly with ultrasonication to prepare 3Dnanofibrous collagen scaffolds with a large surface area for subsequent cell culture studies.

Scanning electron microscopy (SEM) analysis showed that collagen self-assembled into nanofibrous scaffolds with fiber diameters of 100 to 150 $\mathrm{nm}$ and scaffold dimensions in the centimetre range when $\mathrm{pH}$-induced self-assembly was combined with ultrasonication. By controlling the collagen concentration we could reproducibly adjust the scaffold thickness between $500 \mathrm{~nm}$ and $8 \mu \mathrm{m}$. Subsequently, 3T3 mouse fibroblasts were cultivated on nanofibrous collagen with $500 \mathrm{~nm}$ thickness for up to 72 hours. Live-dead staining and cell proliferation assays showed that fibroblasts on collagen nanofibers exhibited a cell viability comparable to planar collagen and glass. Fluorescence microscopy analysis of the cell area revealed that cells on nanofibrous collagen were significantly smaller than on planar collagen or glass. SEM analysis confirmed that the fibroblasts partly grew into the nanofibrous scaffolds (Fig. 1).

Atomic force microscopy studies of the cell stiffness did, however, not yield any significant differences between fibroblasts on collagen nanofibers, planar collagen and glass. We assume that the cell stiffness was mainly influenced by the underlying glass when $500 \mathrm{~nm}$ thick collagen nanofibers were used. Therefore, we will continue studies of the cell mechanics on large-scale collagen scaffolds with a thickness in the micrometre range to establish our nanofibrous collagen 3D-scaffolds as synthetic ECM modification for various biomaterial surfaces.

Fig1: SEM image of 3 T3 fibroblasts growing into nanofibrous collagen

[1] Matthews, J. et al. Biomacromolecules 2002

[2] Harris, J. et al. Micron 2007

[3] Raoufi, M. et al. Integr Biol 2016

Figure 1

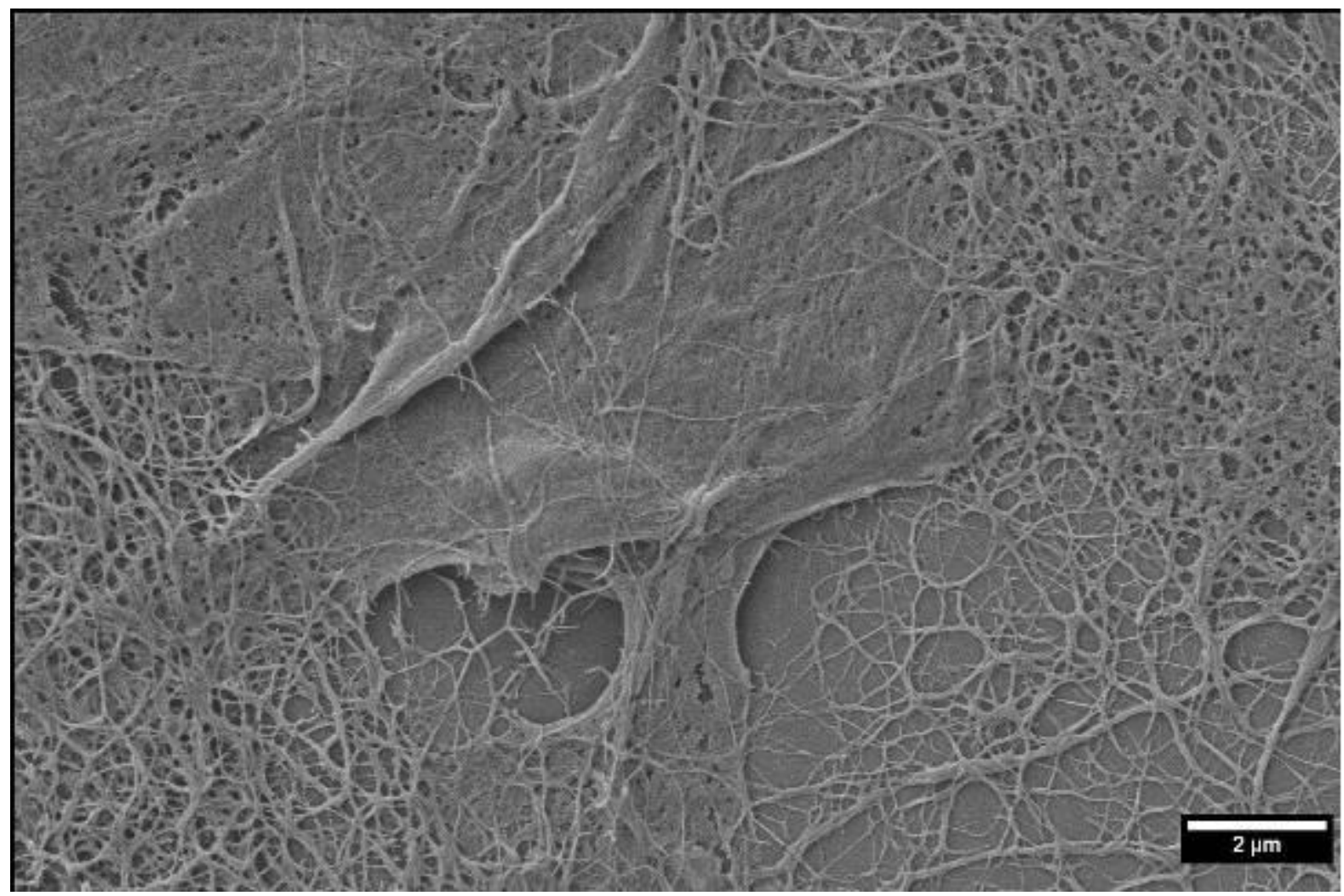


S04-04

\title{
Is there a potential danger of calcium phosphate nanoparticles?
}

\author{
*M. Epple ${ }^{1}$ \\ ${ }^{1}$ University of Duisburg-Essen, Inorganic Chemistry, Essen, Germany
}

Calcium phosphate is applied in many products in biomedicine, but also in toothpastes and cosmetics. In some cases, it is present in nanoparticulate form, either on purpose or after degradation or mechanical abrasion of biomaterials. Concerns have been raised with respect to adverse biological effects of hydroxyapatite nanoparticles, e.g. by the Scientific Committee on Consumer Safety (SCCS) of the European Commission (2015) and in the United States after the discovery of calcium phosphate needles in babyfood (2016). A thorough literature review shows that calcium phosphate nanoparticles have no inherent toxicity but can lead to an increase of the intracellular calcium concentration after endosomal uptake and lysosomal degradation. However, cells are able to clear the calcium from the cytoplasm within a few hours, unless very high doses of calcium phosphate are applied. The observed cytotoxicity in some cell culture studies, mainly for unfunctionalized particles, is probably due to particle agglomeration and subsequent sedimentation onto the cell layer, leading to a very high local particle concentration, a high particle uptake, and subsequent cell death. There is no risk from an oral uptake of calcium phosphate nanoparticles due to their rapid dissolution in the stomach. The risk from dermal or mucosal uptake is very low. Calcium phosphate nanoparticles can enter the bloodstream by inhalation, but no adverse effects have been observed, except for a prolonged exposition to high particle doses. Calcium phosphate nanoparticles inside the body (e.g. after implantation or due to abrasion) do not pose a risk as they are typically resorbed and dissolved by osteoclasts and macrophages. In summary, the risk associated with an exposition to nanoparticulate calcium phosphate in doses that are usually applied in biomedicine, health care products, and cosmetics is shown to be very low and probably not present at all. 


\title{
S04-05
}

\section{biomacs: biomaterials advanced cell screening}

\author{
Q. Zhou ${ }^{1}$, L. Yang ${ }^{1}$, T. van der Boon ${ }^{1}$, L. Ge ${ }^{1}$, C. Guimarães ${ }^{1}$, T. van Kooten ${ }^{1}$, *P. van Rijn ${ }^{1}$
}

${ }^{I}$ University Medical Center Groningen, Biomedical Engineering, Groningen, Netherlands

\section{Introduction}

Biophysical cues located on biomaterial surfaces profoundly affect (stem) cell response, which provides pivotal information for designing biomaterials [1]. However, most of the physicochemical properties were studied individually, which is not appropriate since cells always interact with multiple cues simultaneously.1 We developed an orthogonal double gradient platform to identify the cell response towards thousands of these combined parameters to enable multiparameter investigations (Fig. 1).

Fig. 1: Schematic approach biointerface screening platform

\section{Methods}

PDMS orthogonal double gradients are prepared by sequential shielded air plasma oxidation treatments and chemical modification using silanization reactions in accordance with previously published single linear gradients [2].

\section{Results}

Each position on the orthogonal double gradient surface has a unique combination of stiffness and wettability (stiffness: 6-89 MPa; WCA: $29^{\circ}$ $90^{\circ}$ ). hBM-MSCs were on cultured on the gradient platforms for $24 \mathrm{~h}$, imaged via automated fluorescence microscopy, and quantified for cell response. We found higher cell surface coverage in WCA ranging from $29^{\circ}-36^{\circ}$ and $48^{\circ}-90^{\circ}$ on the soft part (6-19 MPa) of the substrate. However, there is similar cell surface coverage on the stiffer range from 19 to $89 \mathrm{MPa}$ in WCA range from $29^{\circ}$ to $90^{\circ}$. Also hBM-MSC adhesion, spreading, nucleus size and vinculin expression were affected and indicated that hBM-MSC behavior is non-linearly regulated by surface stiffness and wettability.

\section{Conclusion}

We developed a novel approach to elucidate combined physical parameter influences on cellular behaviour. Most cell responses are non-linearly regulated by material stiffness and wettability. Our strategy allows for efficient analysis of multiple cue-response relationships to facilitate enhanced biomaterial development.

\section{References}

[1] A.M. Schaap-Oziemlak, et al., RSC Adv. 4:53307-53320, 2014

[2] Q. Zhou, et al., Adv. Mater. Interfaces, accepted, 2018

\section{Figure 1}

\section{High-Throughput Screening System}
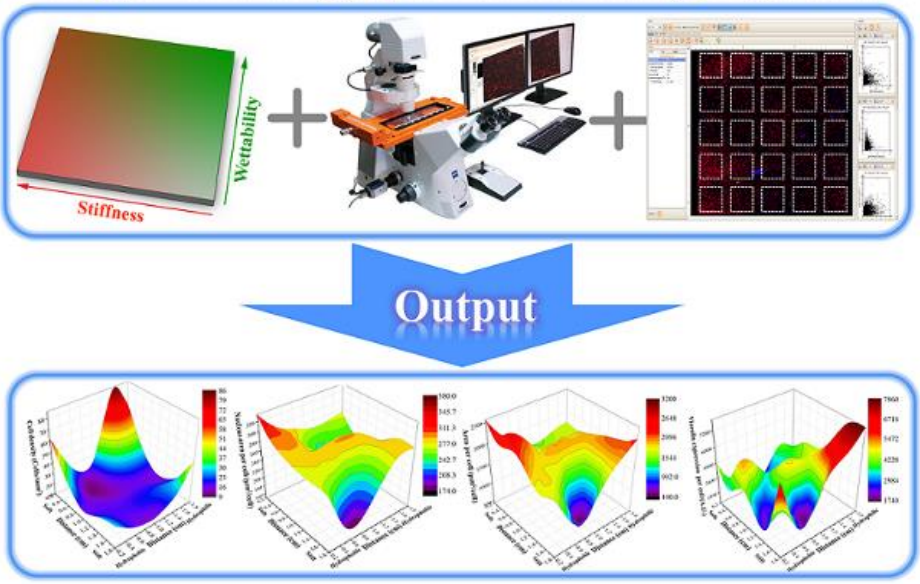


\title{
In vitro co-culture of human osteocytes, osteoclasts and osteoblasts in collagen gels
}

\author{
*A. Bernhardt ${ }^{1}$, J. Skottke ${ }^{1}$, V. Oesterreich ${ }^{1}$, M. Gelinsky ${ }^{1}$ \\ ${ }^{I}$ TU Dresden, Zentrum für Translationale Knochen-, Gelenk- und Weichgewebeforschung, Dresden, Germany
}

Osteocytes have been postulated to orchestrate bone homeostasis by regulating both bone-forming osteoblasts and bone-resorbing osteoclasts. In vitro bone models comprising all three cell types of bone are challenging to establish, since primary osteocytes, which are deeply embedded into the mineralized bone ECM are difficult to isolate, and require different media composition compared to osteoblasts and osteoclasts in vitro. In the present study, co-cultivation of primary osteoblasts, osteocytes and osteoclasts was performed in 3D collagen gels. Osteocytes were isolated from human bone tissue using an optimized protocol of sequential digestion and demineralization. Osteoblasts were obtained from the same bone tissue after short digestion. Osteoclasts were differentiated from human peripheral blood mononuclear cells. After embedding osteocytes into collagen gels osteoblasts and osteoclasts were seeded onto the gel surface and the constructs were co-cultivated for 2 days. Gene expression analysis showed, that typical osteoclast markers tartrate resistant acid phosphatase, cathepsin $\mathrm{K}$ and carbonic anhydrase II were upregulated in co-culture. Furthermore, the dedifferentiation of primary osteocytes, which is observed in single culture, seems to be retarded in co-culture with osteoblasts, since gene expression of osteocyte markers E11/podoplanin and dentin matrix protein 1 was not downregulated in co-culture.

The established in vitro 3D co-culture model will be applied in future for the in vitro testing of drugs, ions, mechanical stimulation as well as release products of different biomaterials for bone regeneration.

\section{Figure 1}
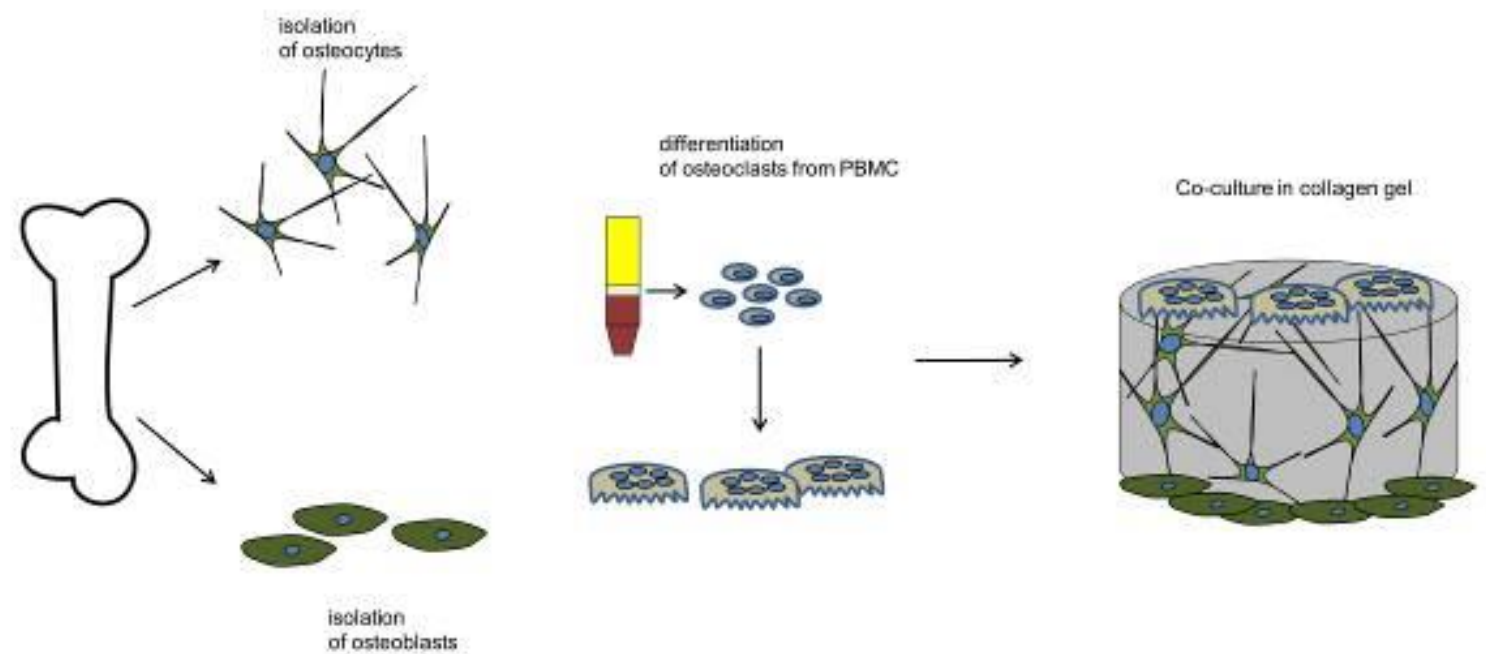